\title{
DEEP LEARNING FOR EPILEPTIC INTRACRANIAL EEG DATA
}

\author{
Andreas Antoniades, Loukianos Spyrou, Clive Cheong Took, and Saeid Sanei \\ Department of Computer Science \\ University of Surrey, Guildford, United Kingdom \\ \{a.antoniades, 1.spyrou, c.cheongtook, s.sanei\}@ surrey.ac.uk
}

\begin{abstract}
Detection algorithms for electroencephalography (EEG) data typically employ handcrafted features that take advantage of the signal's specific properties. In the field of interictal epileptic discharge (IED) detection, the feature representation that provides optimal classification performance is still an unresolved issue. In this paper, we consider deep learning for automatic feature generation from epileptic intracranial EEG data in the time domain. Specifically, we consider convolutional neural networks (CNNs) in a subject independent fashion and demonstrate that meaningful features, representing IEDs are automatically learned. The resulting model achieves state of the art classification performance, provides insights for the different types of IEDs within the group, and is invariant to time differences between the IEDs. This study suggests that automatic feature generation via deep learning is suitable for IEDs and EEG in general.
\end{abstract}

Index Terms - EEG, Deep Learning, Epilepsy, Convolutional Neural Networks.

\section{INTRODUCTION}

Neural networks are a popular class of machine learning algorithms typically used for classification and regression, and have been extensively used in many applications over the past few decades [1]. Considered as blackboxes by many, neural networks create non-linear combinations of the original input to identify its most informative characteristics. Deep learning is possibly one of the most significant advances in neural networks and has found applications mainly in dimensionality reduction and hierarchical multilayer feature learning [2]. Deep neural networks are now considered state of the art algorithms and have won numerous contests in pattern recognition and machine learning, and most importantly can achieve better than human accuracy on classification of benchmark datasets [3].

In a statistical context, deep learning algorithms can be seen as recursive generalised linear models based on regres-

This work has been supported by the EPSRC, UK, Grant No. EP/K005510/1. sion [4]. Statistical methods that employ architectures with one or no hidden layers, perform the so-called shallow learning [5]. On the other hand, deep learning methods make use of architectures of many hidden layers. A number of deep learning algorithms have been proposed over the past years. Stacked Denoising Autoencoders learn useful representations of data by attempting to reconstruct the input signal at each layer while adding artificial noise [6]. Deep Belief Networks operates on stacked Restricted Boltzmann machines and follow a stochastic approach for optimisation [7]. Finally, Convolutional Neural Networks (CNNs) were designed to automatically learn optimal filters that generate high level features when applied to images [8].

Deep learning can benefit electroencephalography (EEG) studies, since neural processes exhibit a hierarchical structure. The electrical response of the brain to a stimulus, task, or physiological state usually comprises of a number of subresponses which are combined to provide the full response. Deep learning has been used for classification in EEG [9], feature extraction [10] and in the epilepsy field for seizure prediction [11]. All these established findings can make sense in the interpretation of EEG signals. This is what our work addresses, making sense of epileptic EEG data without the need for expert domain knowledge.

Interictal epileptiform discharges (IEDs) are transients of electrical activities that appear in EEG recordings of patients with epilepsy. Their accurate detection and localisation is important to the diagnosis and treatment of epilepsy. Many classification algorithms have been developed for the purpose of distinguishing IED from non-IED activity. A review of algorithms is provided in $[12,13]$. The common denominator of all these methods lies in the statistical description of an IED signal, which can be obtained either through modeling or through the use of a similarity-based algorithm. This is often facilitated by obtaining useful representations of the signal that can better exploit its structure. The feature representations that are useful in IED detection has remained a rather unresolved issue [12].

In this work we exploit deep learning to generate an optimal set of features from the EEG signals of 25 patients. We provide a comprehensive analysis on the ways that the CNN- 
based trained filters are suitable for IED feature extraction. That is because IEDs of different patients and channels may exhibit slightly different temporal locations and also in some cases there might be multiple IEDs in a single segment. In Section 2, we provide initial insight on the epileptic dataset and the preprocessing methodology for the intracranial EEG. Section 3 describes in detail the CNN structure and parameters, while Sections 4 and 5 show the results and conclude the paper.

\section{EPILEPTIC EEG}

An epilepsy expert from King's College London Hospital visually inspected the intracranial data and marked the timing information where the intracranial data exhibited visually noticeable epileptic discharges. Classifier training was enabled by slicing the raw EEG data in a $\pm 160 \mathrm{~ms}$ window centered on the intracranial timing scores and baselined on the preceding $160 \mathrm{~ms}$ with the resulting signal finally being linearly detrended to remove undesired drifts. An example IED is provided in Fig. 1. Non-IED segments were also obtained from time segments where there were no scored IEDs. For each subject, the number of sliced IED and non-IED segments was chosen to be the same. The spectrogram method was used to convert the time-domain (TD) signal into time frequency (TF) features with a Hanning-tapered window length of $80 \mathrm{~ms}$ and an overlap of $50 \%$ between windows. The window was chosen so as to capture the morphology of IED spikes whose subcomponents have a duration of less than $80 \mathrm{~ms}$. These TF features were obtained for each IED and non-IED segment. The TD features were of size 12 channels $\times 65$ samples where the TF features were of size 12 channels $\times 7$ frequency bins $\times 9$ time points.

Table 1. Dataset information

\begin{tabular}{cccc}
\hline Subject & No. of trials & Subject & No. of trials \\
\hline 1 & 650 & 14 & 236 \\
2 & 1906 & 15 & 448 \\
3 & 1658 & 16 & 50 \\
4 & 1098 & 17 & 100 \\
5 & 424 & 18 & 146 \\
6 & 1696 & 19 & 76 \\
7 & 398 & 20 & 520 \\
8 & 330 & 21 & 96 \\
9 & 316 & 22 & 46 \\
10 & 684 & 23 & 1212 \\
11 & 682 & 24 & 228 \\
12 & 944 & 25 & 1084 \\
13 & 634 & & \\
\hline No. of segments & & & \\
IED & 9218 & non-IED & 9218 \\
\hline
\end{tabular}




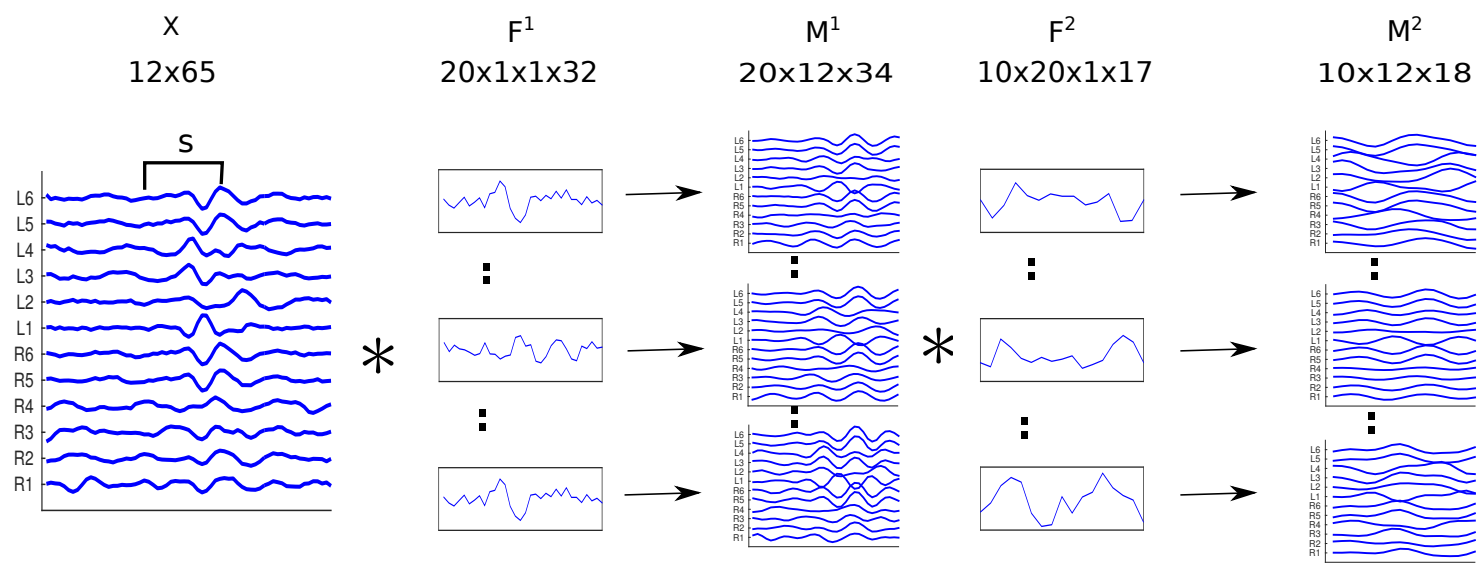

Fig. 2. Convolutional model for EEG signal processing, where $M^{(n)}$ are the feature maps generated at convolutional layer $n, F^{(n)}$ are the filters at layer $n$ and $(*)$ is the convolution operator. M represents a tensor of dimension[Filters $\mathrm{x}$ Channels $\mathrm{x}$ Time].

It can be shown that a number of feature maps can be generated at each layer, by using different sets of weights (filters). Each feature map can detect a specific feature [2].

In the context of EEG, we perform 1-d convolution for each channel separately. 2-d convolution, which is widely used in image processing, is not relevant in this work as the spatial information is lost when considering multichannel EEG data ${ }^{2}$. Each filter sweeps through the data of each channel and produces a new multichannel time series that captures the similarity between EEG and filter. This can be denoted as:

$$
\begin{array}{r}
\left.f \in \text { Filters } \forall c_{n} \text { where }\left\langle c_{n}\right| n \in \text { Channels }\right\rangle \forall s \in c_{n} \\
\text { where } s \in\left(\begin{array}{c}
c_{n} \\
|f|
\end{array}\right) \rightarrow s * f
\end{array}
$$

where $c$ is a sequence representing each channel and $|$.$| is the$ cardinality. This training procedure is depicted in Fig. 2 By using a $\mathrm{CNN}$, we can learn filters that can describe and detect different signal types of EEG data irrespective of their temporal location within the data segment. For each CNN layer, new feature maps are produced (via convolutions) according to the filters of the previous layer. In our proposed algorithm, CNN convolution is performed by convolving each electrode signal with a 1-d filter, adding a bias term and applying a non linear function, in this case hyperbolic tangent.

$$
\begin{gathered}
G_{l, i}^{k}=\sum_{i} F_{j, i}^{k} * M_{l, i}^{k-1}+b_{j, i}^{k} \\
M_{l, j}^{k}=\tanh \left(G_{l, i}^{k}\right)
\end{gathered}
$$

where $*$ is the convolution operator, $F_{j, i}^{k}$ is the $j^{\text {th }}$ filter at layer $k$ for feature map $i, M_{l, j}^{k-1}$ is the $l^{t h}$ feature map at

\footnotetext{
${ }^{2}$ Multichannel EEG data are arbitrarily ordered and the exact spatial information is indicated by the labels of each channel, not the data itself.
}

layer $k-1, M_{l, i}^{k}$ are the feature maps at layer $k$, generated from the convolution of filters at layer $k$ and the feature maps of layer $k-1$ and $b^{(k)_{j, i}}$ is the bias term at layer $k$ for filter $F_{j, i}^{k}$. The hyperbolic tangent function is defined as $\tanh (\theta)=\frac{\sinh (\theta)}{\cosh (\theta)}=\frac{\exp ^{\theta}-\exp ^{-\theta}}{\exp ^{\theta}+\exp ^{-\theta}}$. This way, deep layers can learn complex features as combinations of filters from the preceding layers. For clarity, we include the derivation of backward propagation for our model. Given an error function $E$, the gradient component is computed by calculating the partial derivative $\frac{\partial E}{\partial F_{j, i}}$ with the use of the chain rule.

$$
\frac{\partial E}{\partial F_{j, i}}=\sum_{i} \frac{\partial E}{\partial G_{l, i}^{k}} \frac{\partial G_{l, i}^{k}}{\partial F_{j, i}}=\sum_{i} \frac{\partial E}{\partial G_{l, i}^{k}} M_{l, i}^{k-1}
$$

We can then calculate the partial derivative of the error with respect to the output of each neuron, $\frac{\partial E}{\partial G_{l, i}^{k}}$, to compute the gradient for the current layer.

$$
\begin{aligned}
\frac{\partial E}{\partial G_{l, i}^{k}} & =\frac{\partial E}{\partial M_{l, i}^{k}} \frac{\partial M_{l, i}^{k}}{\partial G_{l, i}^{k}} \\
& =\frac{\partial E}{\partial M_{l, i}^{k}} \frac{\partial \tanh \left(G_{l, i}^{k}\right)}{\partial G_{l, i}^{k}} \\
& =\frac{\partial E}{\partial M_{l, i}^{k}} \tanh ^{\prime}\left(G_{l, i}^{k}\right)
\end{aligned}
$$

Finally we back propagate the errors to the previous layers.

$$
\frac{\partial E}{\partial M_{l, i}^{k-1}}=\sum_{i} \frac{\partial E}{\partial G_{l, i}^{k}} \frac{\partial G_{l, i}^{k}}{\partial M_{l, i}^{k-1}}=\sum_{i} \frac{\partial E}{\partial G_{l, i}^{k}} F_{j, i}^{k}
$$

Note that, although Eq.(1) indicates the need of summation in two dimensions, we only employ a 1-D filter, as seen in Eq(3), and therefore require summation across one dimension. For 
the purposes of this paper the cross entropy error function $E=-\frac{1}{N} \sum_{j=1}^{N} x(n) \log (y(n))+(1-x(n)) \log (1-y(n))$ is considered.

\subsection{Training procedure}

We have used the leave-subject-out method for our experiments. This method uses the data of a subject for testing and the other 24 for training. This procedure is repeated until all subjects are used for testing. Initial experimentation has revealed that using very deep networks of more than two convolutional layers, does not improve the classification rate. Two CNN topologies were considered, CNN1 comprised of a single convolution layer whereas CNN2 comprised of two convolutional layers. Both networks were followed by a single fully-connected sigmoidal hidden layer and a logistic regression model to perform classification on the generated features. The network was trained using backpropagation until convergence. The parameters for the two CNNs are summarised in Table 2. We compare the proposed CNN architecture with

Table 2. Training parameters for the all the considered methods

\begin{tabular}{lcccc}
\hline Parameter/Method & TD [13] & TF [13] & CNN1 & CNN2 \\
\hline Convolution layers & - & - & 1 & 2 \\
No. of filters & - & - & 126 & 20,10 \\
Filter order & - & - & 32 & 32,17 \\
Sigmoidal layer size & - & - & 500 & 500 \\
\hline Network parameters & - & - & 4532 & 4540 \\
Classifier features & 780 & 756 & 500 & 500 \\
\hline
\end{tabular}

a state-of-the-art classification algorithm [13] that only uses a single logistic regression layer but with handcrafted optimised features. State-of-the-art performance is achieved with TF features (see Section 2) with a logistic regression classifier. We include four comparisons in this study: TD features with logistic regression, TF with logistic regression, and CNN1/CNN2 with TD features. Table 3 summarises the classification accuracy of each method.

\section{MAKING SENSE OF EPILEPTIC EEG IN MACHINE LEARNING}

To address the 'black-box' nature of neural networks, we now illustrate how the learning process of CNNs captures features of IEDs. Fig. 3 shows the evolution of learning weights pertaining to the first layer of CNN2 through training and how it correlates with an averaged IED. The corellation of layer 1 reaches $42 \%$ at convergence. We must note that this correlation is between a single filter and the average of all IEDs. At the second layer a correlation of $52 \%$ is observed. Inspecting Fig. 4, it is clear that from the top-left most subplot to the bottom-right most subplot that these learning coefficients are converging towards the waveform of an IED, as shown previously in Fig 1. Also, notice that two epileptic spikes are captured through the weights.

In, Fig. 4 the first layer filters of CNN2 (leftmost subplots) are compared with its second layer convolved filters (rightmost subplots). Observe that the background EEG occurs at the start of each subplot, followed by the occurrence of epileptic spikes, which are then followed again by background EEG. The second layer learning coefficients (rightmost subplot) captured more complex and well-defined epileptic shapes than those of first layer (leftmost subplots). In other words, background EEG is made much more distinct from those spikes. This enhanced distinction between background activity and epileptic spikes should improve the detection of those spikes for classification from shallow learning (CNN1) to deep learning (CNN2), as confirmed by the classification accuracy provided in Table 1.

The left plot of Fig. 5 illustrates the inputs to layer 1 of the IED class, whereas that of Fig. 6 shows the inputs to layer 1 pertaining to the non-IED class. On the other hand, the right plots of both figures show the outputs of the first layer, resulting from the convolution with the learnt filters in Fig. 4 (leftmost subplots) depicting epileptic waveforms. Notice that the high magnitude activations in the output signals in Fig. 5 is much more synchronised in the neighbouring electrodes than those in Fig. 6. This again indicates that the epileptic patterns were successfully learnt by our proposed method.

Although shallow learning of CNN1 yielded poorer results than those in our previous work [13], deep learning of CNN2 had similar performances as in [13]. This was confirmed by McNemars statistical test to assess the significance difference between the CNN1 method and the other three. TD-CNN2 and CNN1-CNN2 had significant difference with $p<0.01$, while TF-CNN2 was not significant. In other words, our approach CCN2 provided similar performance results as those in our previous work [13]. Yet, the advantage of the methodology proposed herein was to circumvent the use of time-frequency analysis, facilitating the interpretation of the EEG data.

\section{CONCLUDING REMARKS}

We have demonstrated that CNNs can learn the intracranial IEDs waveform patterns. This has important healthcare implications as the shape of the IED can assist the diagnosis of epilepsy. The results of this paper are instructive and suggest that automatic feature generation based on deep learning is a potentially useful tool for IED detection and EEG data in general. Future works include deeper learning with additional hidden layers in CNNs while minimising information loss and a closer examination of the different kinds of epileptic waveforms in neural network learning. 

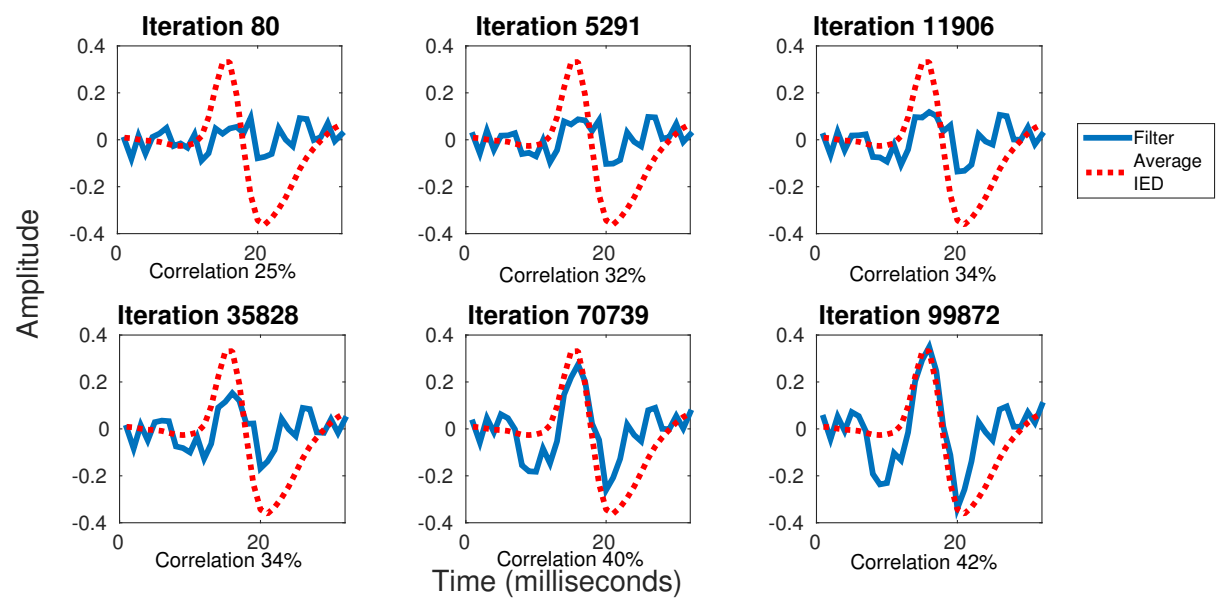

Fig. 3. Evolution of learnt coefficients towards the morphology of an epileptic waveform. Correlation does not exceed $42 \%$ as we are comparing a single filter with the average of different IEDs.
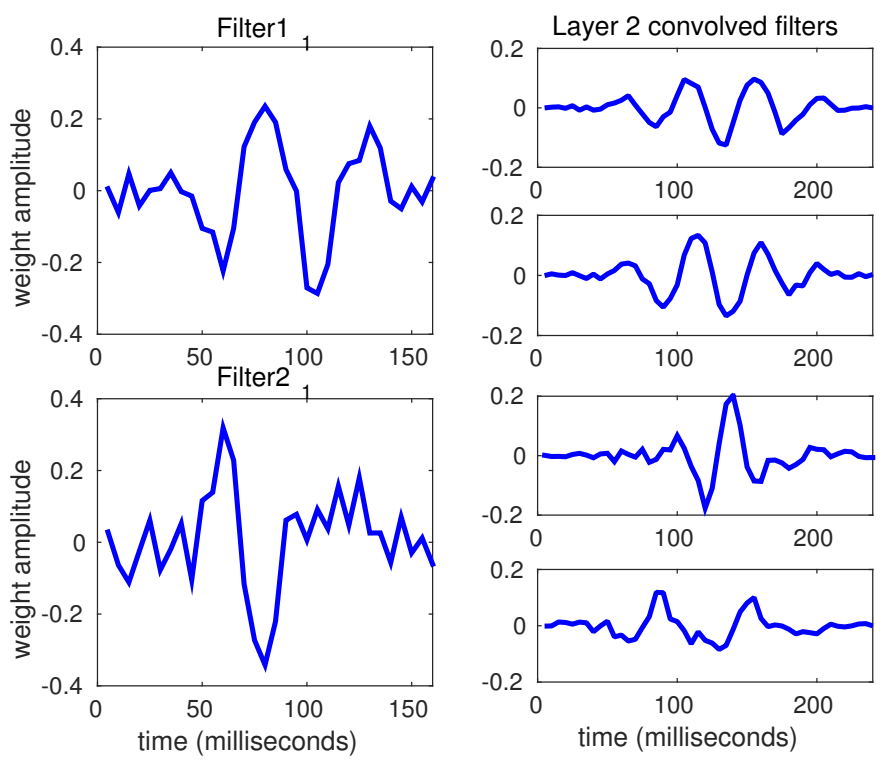

Fig. 4. Learnt filter coefficients for layer 1 for simple IED waveforms (left) and convolved learnt filters for both layers for more complex shapes (right).
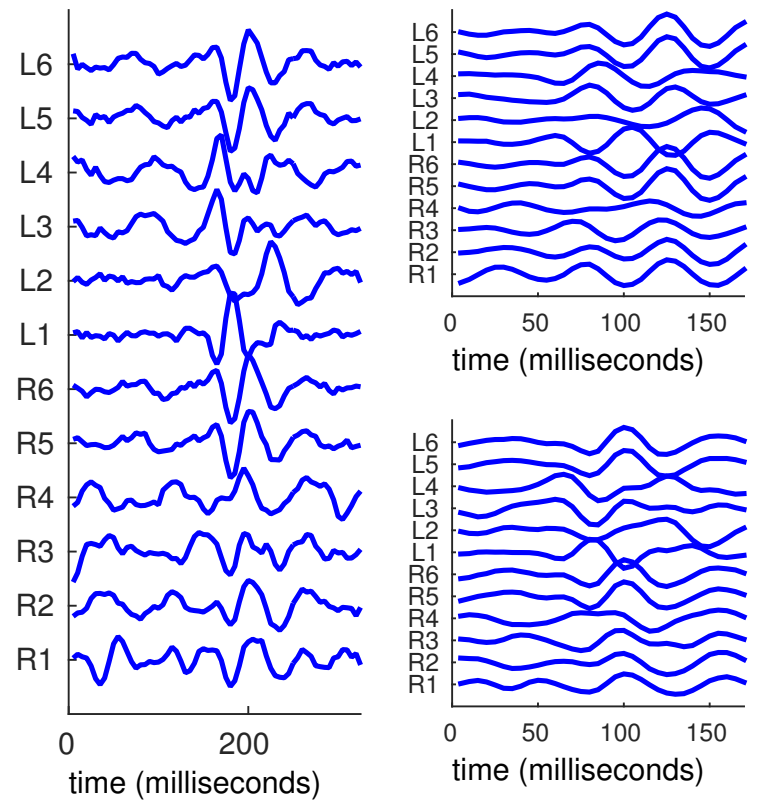

Fig. 5. Example segment of the IED class and its layer 1 feature map (multichannel) for the two filters shown on the L.H.S of Fig. 4. 


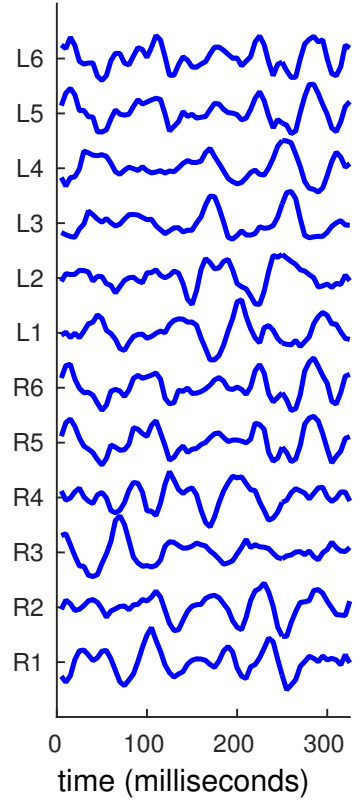

Fig. 6. Example segment of the non-IED class and its layer 1 feature map (multichannel) for the two filters of Fig. 4.

\section{REFERENCES}

[1] K. Hornik, M. Stinchcombe, and H. White, "Multilayer feedforward networks are universal approximators", in Neural Networks, vol.2, pp. 359-366, 1989.

[2] G. Hinton, R. Salakhutdinov, "Reducing the dimensionality of data with neural networks", Science Magazine, vol 313, pp. 504-507, 2006.

[3] J. Schmidhuber, "Deep learning in neural networks: An overview", arXiv preprint arXiv:1404.7828, 2014

[4] A. Davison, C., "Statistical models", Cambridge series in statistical and probabilistic mathematics. vol. 11, Cambridge: Cambridge University press, 2008.

[5] N.G. Polson, M.Heidari and B. Willard, "A Statistical Theory of Deep Learning via Proximal Splitting”, arXiv 1509.06061v1, 2015.

[6] P. Vincent, H. Larochelle, I. Lajoie, Y. Bengio, and P.-A. Manzagol, "Stacked denoising autoencoders: Learning useful representations in a deep network with a local denoising criterion", in Proceedings of the 27th International Conference on Machine Learning, pp. 33713408, ACM, 2010.

[7] G.E. Hinton, S. Osindero, and Y. Teh, "A fast learning algorithm for deep belief nets", Neural Computation, pp. 1527-1554, 2006.

[8] D. C. Ciresan, U. Meier, J. Masci, L. M. Gambardella and J.Schmidhuber, "Flexible, high performance convolutional neural networks for image classification", in IJCAI, pp. 1237-1242, 2001.

[9] Wulsin DF, Gupta JR, Mani R, Blanco JA, Litt B. "Modeling electroencephalography waveforms with semi-supervised deep belief nets: fast classification and anomaly measurement”, J. Neural Eng. 8(3):036015, Jun. 2011.

[10] R. Yuanfang and Y. Wu, "Convolutional deep belief networks for feature extraction of EEG signal", International Joint Conference on Neural Networks (IJCNN), Beijing, China, 2014.

[11] P.W. Mirowski, Y. LeCun, D. Madhavan, R. Kuzniecky, "Comparing SVM and convolutional networks for epileptic seizure prediction from intracranial EEG" in Machine Learning for Signal Processing, pp.244-249, 16-19 Oct. 2008.
Table 3. Classification accuracy for different approaches (\%)

\begin{tabular}{ccccc}
\hline Subject & Temporal & Time-Frequency & CNN1 & CNN2 \\
\hline 1 & 78.92 & 83.54 & 81.70 & 87.54 \\
2 & 89.83 & 89.75 & 78.55 & 87.31 \\
3 & 83.37 & 89.75 & 78.59 & 85.53 \\
4 & 69.24 & 88.18 & 77.42 & 84.80 \\
5 & 70.19 & 88.81 & 78.31 & 89.85 \\
6 & 81.37 & 91.04 & 85.48 & 90.73 \\
7 & 76.00 & 89.68 & 81.91 & 86.69 \\
8 & 77.89 & 85.68 & 82.13 & 90.91 \\
9 & 80.91 & 88.79 & 79.12 & 90.19 \\
10 & 67.72 & 87.66 & 83.34 & 90.94 \\
11 & 75.73 & 91.52 & 81.19 & 88.27 \\
12 & 79.03 & 87.83 & 80.00 & 89.62 \\
13 & 76.91 & 91.00 & 82.18 & 89.12 \\
14 & 80.80 & 92.41 & 80.81 & 90.85 \\
15 & 89.83 & 89.75 & 82.00 & 92.00 \\
16 & 94.00 & 89.00 & 84.00 & 93.00 \\
17 & 85.21 & 94.37 & 82.88 & 92.47 \\
18 & 61.27 & 84.00 & 71.05 & 88.16 \\
19 & 49.62 & 47.12 & 50.39 & 50.43 \\
20 & 84.37 & 87.50 & 81.25 & 86.31 \\
21 & 59.01 & 81.37 & 84.79 & 91.31 \\
22 & 83.58 & 88.61 & 78.80 & 87.30 \\
23 & 68.86 & 91.23 & 80.27 & 87.29 \\
24 & 86.80 & 81.35 & 75.47 & 85.75 \\
25 & 79.66 & 88.98 & 86.45 & 92.83 \\
\hline Mean & 77.20 & 86.67 & 79.48 & 87.51 \\
\hline & & & &
\end{tabular}

[12] T. Alexandros, M. Tsipouras, D. Tsalikakis , E. Karvounis, L. Astrakas, S. Konitsiotis and M. Tzaphlidou, "Automated epileptic seizure detection methods : a review study" Epilepsy - Histological, Electroencephalographic and Psychological Aspects, 2012.

[13] L. Spyrou, D. Lopez, A. Valentin, G. Alarcon, S. Sanei. "Detection of Intracranial Signatures of Interictal Epileptiform Discharges from Concurrent Scalp EEG.”,DOI: 10.1142/S0129065716500167, International Journal of Neural Systems, 2016. 